\title{
High-resolution Millimeter Imaging of the CI Tau Protoplanetary Disk: A Massive Ensemble of Protoplanets from 0.1 to 100 au
}

\author{
C. J. Clarke ${ }^{1}\left(\mathbb{D}\right.$, M. Tazzari ${ }^{1}$ (1), A. Juhasz ${ }^{1}$, G. Rosotti $^{1}$, R. Booth ${ }^{1}$, S. Facchini ${ }^{2}(\mathbb{D}$,

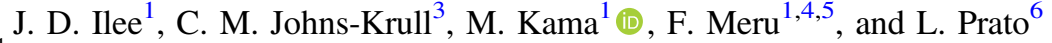 \\ 1 Institute of Astronomy, Madingley Road, Cambridge CB3 OHA, UK; cclarke@ ast.cam.ac.uk \\ ${ }^{2}$ Max-Planck-Institut für Extraterristische Physik, Giessenbachstrasse 1, D-85748 Garching, DE, Germany \\ ${ }_{3}$ Physics \& Astronomy Dept., Rice University, 6100 Main Street, Houston, TX 77005, USA \\ ${ }^{4}$ Department of Physics, University of Warwick, Gibbet Hill Road, Coventry CV4 7AL, UK \\ ${ }^{5}$ Centre for Exoplanets and Habitability, University of Warwick, Gibbet Hill Road, Coventry CV4 7AL, UK \\ ${ }^{6}$ Lowell Observatory, 1400 West Mars Hill Road, Flagstaff, AZ 86001, USA \\ Received 2018 August 9; revised 2018 September 19; accepted 2018 September 21; published 2018 October 4
}

\begin{abstract}
We present high-resolution millimeter continuum imaging of the disk surrounding the young star CI Tau, a system hosting the first hot Jupiter candidate in a protoplanetary disk system. The system has extended mm emission on which are superposed three prominent annular gaps at radii $\sim 13,39$, and 100 au. We argue that these gaps are most likely to be generated by massive planets so that, including the hot Jupiter, the system contains four gas giant planets at an age of only 2 Myr. Two of the new planets are similarly located to those inferred in the famous HL Tau protoplanetary disk; in CI Tau, additional observational data enables a more complete analysis of the system properties than was possible for HL Tau. Our dust and gas dynamical modeling satisfies every available observational constraint and points to the most massive ensemble of exoplanets ever detected at this age, with its four planets spanning a factor 1000 in orbital radius. Our results show that the association between hot Jupiters and gas giants on wider orbits, observed in older stars, is apparently in place at an early evolutionary stage.
\end{abstract}

Key words: planet-disk interactions - protoplanetary disks - submillimeter: planetary systems

\section{Introduction}

Since the 1995 discovery of the first hot Jupiter (Mayor \& Queloz 1995), it is now established that such gas giant planets orbiting at radii $<0.1$ au from their parent stars are found in around $1 \%$ of main-sequence solar-type stars (Wright et al. 2012). There is considerable debate as to whether these objects formed in situ or have instead migrated from larger radii, either from interaction with their natal protoplanetary disk (Kley \& Nelson 2012) or planet-planet scattering after the disk has dispersed (Rasio \& Ford 1996). With typical ages of up to several Gyr, most hot Jupiter hosts have long since lost their protoplanetary disks (typical lifetime of a few Myr; Haisch et al. 2001); arguments about the origin of hot Jupiters are thus usually based on theoretical models linking hypothetical initial conditions to present-day orbital parameters.

The recent discovery (Johns-Krull et al. 2016; Biddle et al. 2018), using the radial velocity technique, of a hot Jupiter in the young disk-bearing solar-type star CI Tau, has demonstrated that in at least this case the hot Jupiter is already in a very close orbit when the star is only $\sim 2$ Myr old (Guilloteau et al. 2014). CI Tau is a well-studied system, with mass $0.92 M_{\odot}$ (Simon et al. 2017), luminosity $0.93 L_{\odot}$ (Guilloteau et al. 2014), and is already known to host a massive dust and gas disk extending many hundreds of au from the star (Andrews \& Williams 2007; Guilloteau et al. 2011); additionally it displays a high accretion rate of gas onto the star (McClure et al. 2013). The hot Jupiter's mass is $\sim 11.3 M_{\text {Jupiter }}$ if its orbit is aligned with the outer disk (Guilloteau et al. 2014), consistent with the orbital alignment between hot Jupiters and outer planets found in mature exoplanetary systems (Becker et al. 2017). This mass places it in the top 5\% of the mainsequence hot Jupiter population. Around half of mature hot
Jupiter systems also contain companions (Knutson et al. 2014; Ngo et al. 2015) at less than 20 au which, if present at early times, would create structure in the protoplanetary disk. Although previous submillimeter observations have hinted at a possible gap in the disk around CI Tau at $100 \mathrm{au}$, they lacked the resolution to characterize this in detail or probe the inner disk where companions may be expected (Konishi et al. 2018).

Here we present high-resolution $1.3 \mathrm{~mm}$ Atacama Large Millimeter/submillimeter Array (ALMA) imaging of the disk surrounding CI Tau and report three pronounced annular gaps in emission between 10 and 100 au. We present visibility modeling and explore the origin of these structures via hydrodynamical modeling, arguing for the presence of three gas giants as outer companions to the hot Jupiter.

\section{Observations}

CI Tau was observed with ALMA on 2017 September 23 and 24 (Project ID: 2016.1.01370.S, PI: C. J. Clarke) with 40 antennas (baselines between 21 and $12145.2 \mathrm{~m}$ ) and an onsource integration time of 32.35 minutes in both cases. The correlator used four spectral windows centered at 224, 226, 240 , and $242 \mathrm{GHz}$ in time division mode to measure the continuum in Band 6. Each spectral window used 128 channels and a bandwidth of $1.875 \mathrm{GHz}$, together providing an effective total continuum bandwidth of $7.5 \mathrm{GHz}$. To calibrate the visibilities, a set of standard calibrators were also observed. Calibration of the complex interferometric visibilities used the Common Astronomy Software Applications (CASA) v5.1.1 and the ALMA Pipeline. In panel (a) of Figure 1 we imaged the calibrated visibilities using the multi-scale CLEAN algorithm

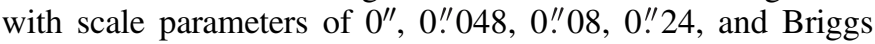
weighting with a robust parameter of 0.5 to obtain the optimal 


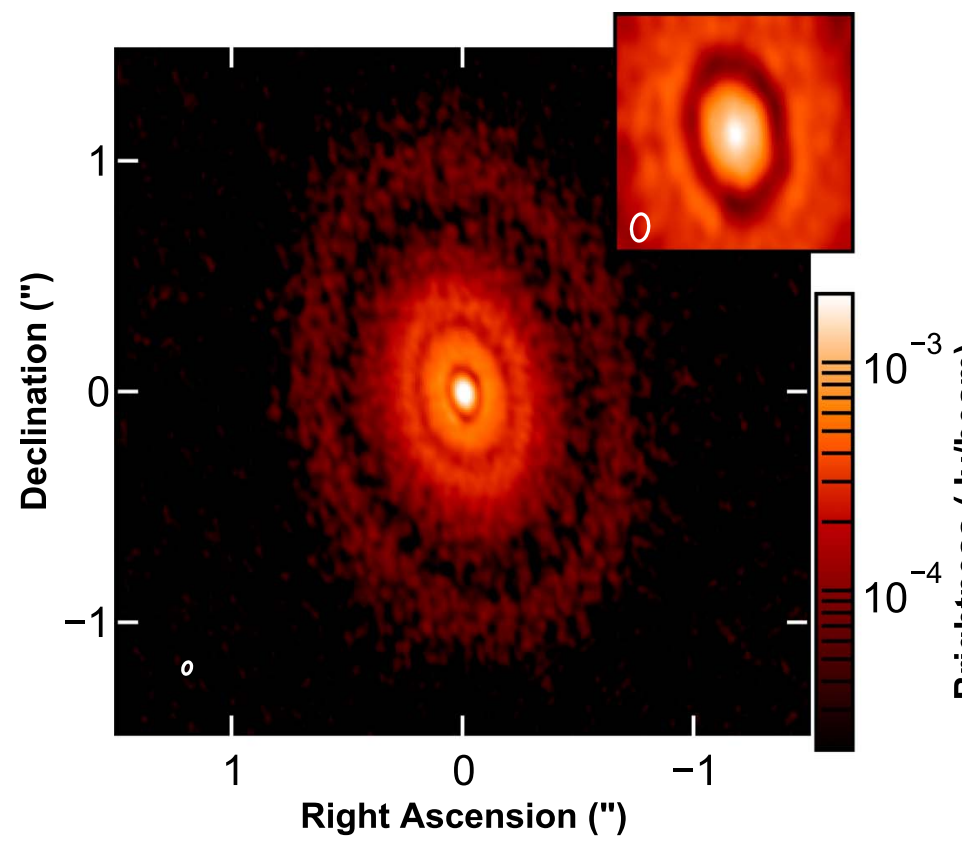

(a)

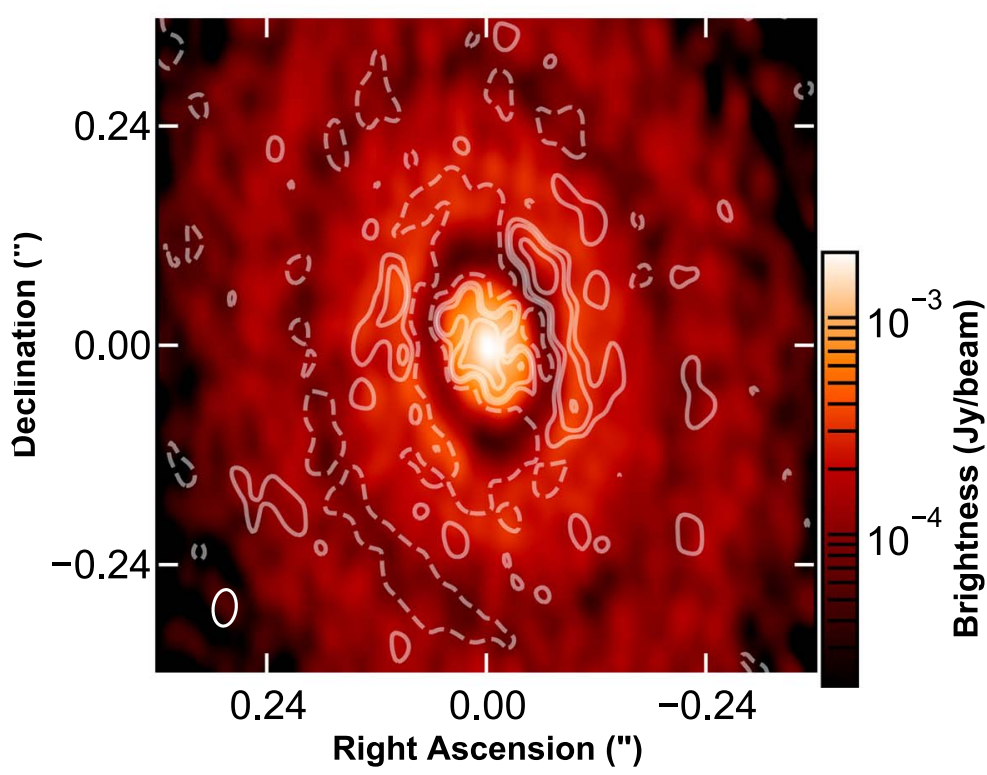

(c)

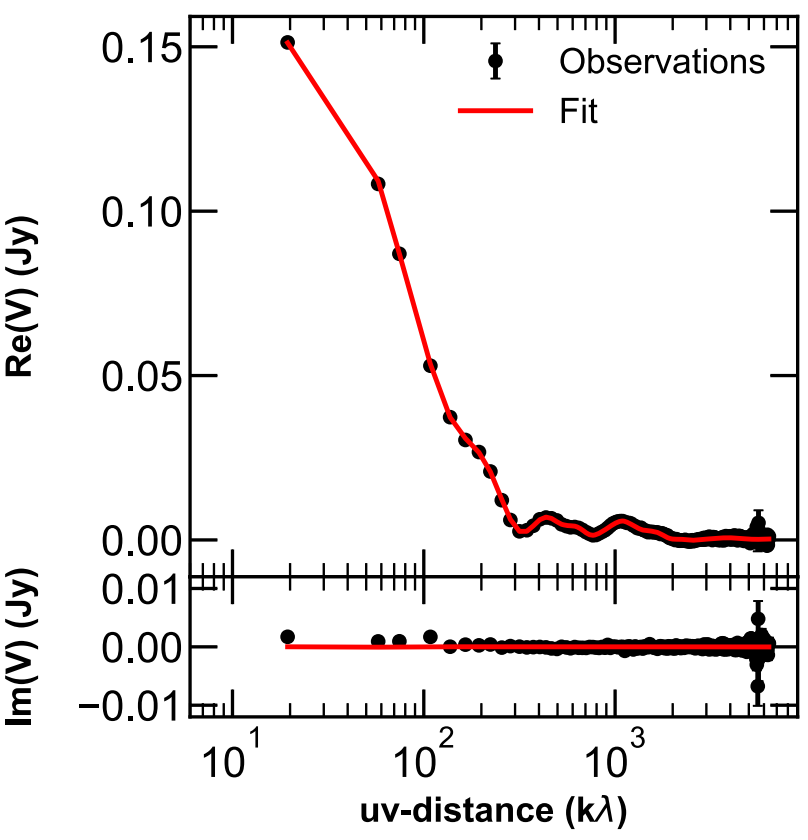

(b)

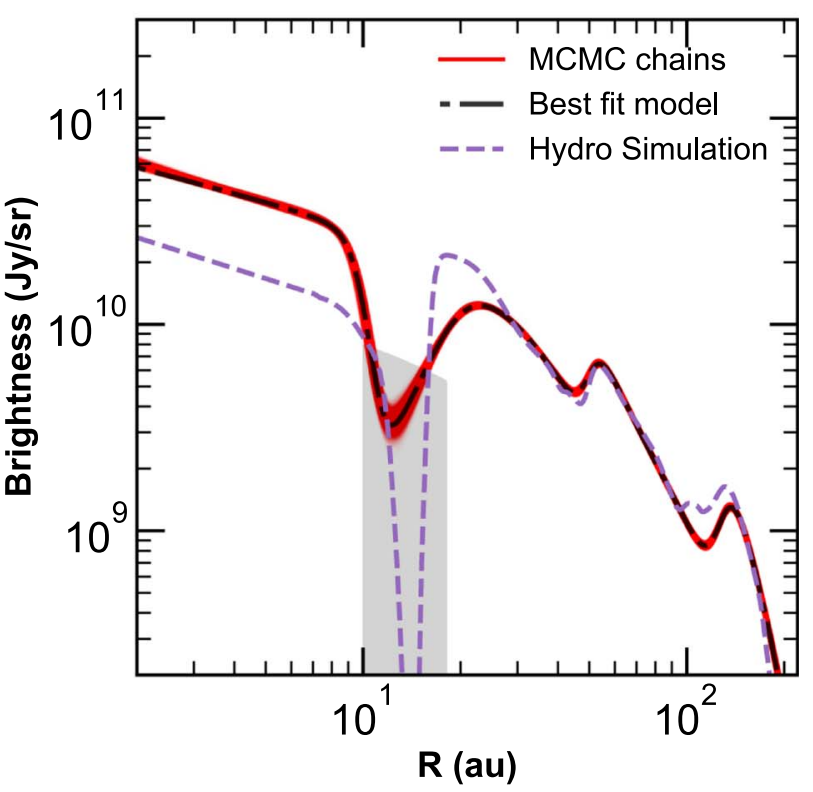

(d)

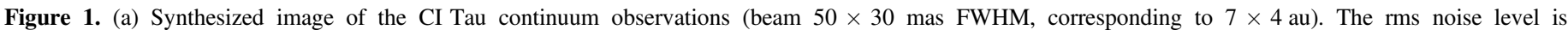

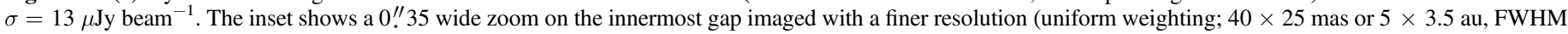

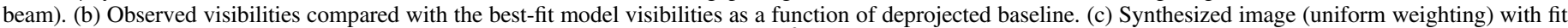

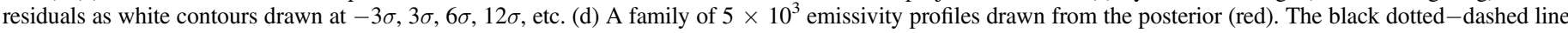

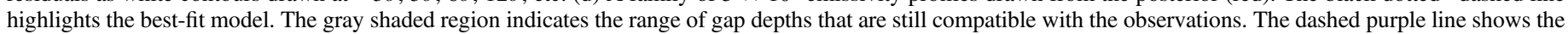
brightness profile obtained from the hydrodynamical simulation.

signal-to-noise ratio $(\mathrm{S} / \mathrm{N})$ and spatial resolution. The resulting synthesized beam is 0 ! $05 \times 0$ ! 03 with a position angle of $16^{\circ}$, while the achieved rms noise level is $13 \mu \mathrm{Jy}^{\text {beam }^{-1}}$.

\section{Visibility Modeling}

We characterize the CI Tau brightness by fitting the continuum visibilities with an axisymmetric parametric model consisting of an envelope and three gaps. For the envelope we use an exponentially tapered power law

$$
I_{\text {env }}(R)=I_{0}\left(\frac{R}{R_{c}}\right)^{\gamma_{1}} \exp \left[-\left(\frac{R}{R_{c}}\right)^{\gamma_{2}}\right] \text { for } R \leqslant R_{\text {out }}
$$

with $I_{0}$ a brightness normalization constant $\left(\mathrm{Jy} \mathrm{sr}^{-1}\right)$. Each gap is parametrized as the difference of two logistic functions:

$$
I_{\text {gap }}(R)=\delta_{\text {gap }}\left[\frac{1}{1+e^{-k_{2}\left(R-R_{\mathrm{g}}-w_{2}\right)}}-\frac{1}{1+e^{-k_{1}\left(R-R_{\mathrm{g}}+w_{1}\right)}}\right],
$$


where $\delta_{\text {gap }}$ describes the gap depth $\left(\delta_{\text {gap }}=0\right.$ corresponding to no gap), $R_{\mathrm{g}}$ is the gap radial location, $w_{1}$ and $w_{2}$ are the leftand right-hand gap widths at half depth and $k_{1}, k_{2}$ express the steepness of the left and right gap profile. The brightness profile is given by

$$
I(R)=I_{\mathrm{env}}\left(1+I_{\mathrm{gap}, 1}\right)\left(1+I_{\mathrm{gap}, 2}\right)\left(1+I_{\mathrm{gap}, 3}\right),
$$

involving five free parameters for $I_{\text {env }}$ and six free parameters for each $I_{\text {gap }}$. We simultaneously fit the disk inclination $i$ and position angle (PA; defined east of north) and the offset $(\Delta$ R.A., $\Delta$ decl.) from the phase center. We thus have a parameter set $\theta=\left(I_{0}, R_{c}, \gamma_{1}, \gamma_{2}, \ldots\right)$ described by 23 parameters for the brightness profile plus four for the system geometry. The computation of the visibilities $V_{\text {mod }}$ for each model $\theta$ is performed using GALARIO ${ }^{7}$ (Tazzari et al. 2018), which first computes the $2 \mathrm{D}$ image of the disk for a given $I(R)$ and then Fourier transforms and samples it in the observed $(u, v)$ points. The likelihood of the observations $V_{\text {obs }}$ given the model visibilities $V_{\bmod }(\theta)$ is assumed Gaussian:

$$
\log p\left(V_{\mathrm{obs}} \mid \theta\right)=-\frac{1}{2} \chi^{2}=-\frac{1}{2} \sum_{j=1}^{N}\left|V_{\mathrm{mod}}-V_{\mathrm{obs}}\right|^{2} w_{j}
$$

where $N$ is the total number of visibility points and $w_{j}$ is the weight ${ }^{8}$ of the $j$ th visibility. The parameter space is explored with a Bayesian approach using the EMCEE Markov Chain Monte Carlo (MCMC) ensemble sampler (Foreman-Mackey et al. 2013), providing an estimate of the posterior probability distribution of the model parameters given the observations

$$
\log p\left(\theta \mid V_{\text {obs }}\right)=\log p\left(V_{\text {obs }} \mid \theta\right)+\log p(\theta)+C
$$

where $p(\theta)$ is the prior on the parameters, and $C$ is a normalization constant. Because the parameters are independent, the priors can be written as $p(\theta)=\prod_{i} p\left(\theta_{i}\right)$. We choose uniform priors on all parameters, except for the inclination for which $p(i)=\sin (i)$ for $0 \leqslant i \leqslant \pi / 2$.

We run the MCMC sampler with 120 walkers for $50 \times 10^{3}$ steps after a burn in phase of $30 \times 10^{3}$ steps. We assessed convergence through visual inspection of the chains' trace plots and also by estimating the autocorrelation time (Foreman-Mackey et al. 2013), resulting in $\sim 150$ steps on average for all parameters. From the $6 \times 10^{6}$ samples in the MCMC chain, we select as bestfit model the maximum likelihood model, i.e., that with lowest normalized $\chi^{2} \simeq 1$, as given by the following parameters: $I_{0}=10.72 \mathrm{Jy} \mathrm{sr}^{-1}, R_{c}=0$ ". $46, \gamma_{1}=-0.39, \gamma_{2}=1.50, R_{g, 1}=$ 0 ". 12, $w_{1 g, 1}=0$ ". $05, \quad w_{2 g, 1}=0$ ". $01, \quad k_{1 g, 1}=222.04 \operatorname{arcsec}^{-1}$, $k_{2 g, 1}=52.82 \operatorname{arcsec}^{-1}, \delta_{g, 1}=0.98, R_{g, 2}=0$ "' $25, w_{1 g, 2}=0$ ". 29 , $w_{2 g, 2}=0$.'11, $k_{1 g, 2}=95.08 \operatorname{arcsec}^{-1}, \quad k_{2 g, 2}=59.77 \operatorname{arcsec}^{-1}$, $\delta_{g, 2}=0.70, R_{g, 3}=0 . " 88, w_{1 g, 3}=0 . \prime 64, w_{2 g, 3}=0$. . $06, k_{1 g, 3}=$ $11.68 \operatorname{arcsec}^{-1}, k_{2 g, 3}=21.87 \operatorname{arcsec}^{-1}, \delta_{g, 3}=0.84, R_{\text {out }}=2 ! .77$, $i=49^{\circ} .24, \mathrm{PA}=11.28, \Delta$ R.A. $=0$ " $33, \Delta$ decl. $=-0$ " 09 . This maximum likelihood model falls in the central $68 \%$ interval of the posterior distribution of all parameters and, indeed, its brightness

\footnotetext{
https://github.com/mtazzari/galario

8 The visibility weights $w_{j}$ are the theoretical estimates obtained by the CASA software package.
}

profile is representative of the density of models generated by the posterior (see panel (d) of Figure 1).

In panel (b) of Figure 1 we compare the observed visibilities and those of the best-fit model as a function of deprojected baseline. Panel (d) shows a family of $5 \times 10^{3}$ models drawn from the inferred posterior (red lines) and the best-fit model (black dotted-dashed line): assuming a distance of $140 \mathrm{pc}$ (Guilloteau et al. 2014), the brightness profile is tightly constrained between 20 and 100 au (i.e., the spatial scales probed by most of the interferometric baselines in the data set) and more uncertain for $R<20$ au. Thus we cannot firmly constrain the detailed shape of the innermost gap, whose width is comparable to the beam $(\sim 7 \mathrm{au})$. We explored in greater detail this degeneracy with a dedicated model suite and found an upper limit on the ratio of the flux inside to outside the gap of $~ 0.28$ : the gray shaded area in panel (d) highlights the range of brightness values that is compatible with the data.

The synthesized image of the residuals obtained for the bestfit model is shown in panel (c) of Figure 1: there are virtually no residuals $(<3 \sigma)$ in most of the disk at radii $R>25$ au, confirming the axisymmetry of the brightness profile. The residuals are most significant (up to a $12 \sigma$ level) at the disk center and in the north-west of the innermost ring. The central residuals reflect the fact that the functional form that we have adopted is insufficiently flexible to correctly capture the emissivity profile in the innermost disk. The latter nonaxisymmetric residuals might be caused by a combination of optical depth effects owing to the viewing angle of the observations $\left(i \sim 49^{\circ}\right)$ and a genuine difference in the local dust temperature.

We note that any perturbation of the disk caused by the hot Jupiter at 0.1 au would occur on a scale of a few times its orbital radius and would thus be indistinguishable within the synthesized beam of $7 \times 4$ au.

\section{Modeling the Emissivity Profile: Evidence of Multiple Planets?}

Structure in protoplanetary disks can derive from many causes. The non-planetary mechanisms that have been proposed to date, however, are not well matched to CI Tau (e.g., photoevaporation produces holes rather than gaps; Ercolano et al. 2017), while simulations of the vertical shear instability (Flock et al. 2017) and of non-ideal magnetohydrodynamic (MHD) effects (Flock et al. 2015) do not produce the well-spaced multiple rings seen in CI Tau. While gaps may also arise from opacity effects associated with ice sublimation fronts (Zhang et al. 2016), the outermost two rings are well outside of the sublimation fronts of even the least volatile species, $\mathrm{N}_{2}$ and CO (Kwon et al. 2015). Moreover, in the innermost gap, our modeling implies a depletion of the optical depth by a factor $\sim 50$ compared with adjacent regions, considerably more than can be attributed to opacity variations. We therefore focus on the planetary hypothesis.

While gap width can be used to infer the required planet mass (Rosotti et al. 2016), this conversion depends on the turbulence in the disk, as parameterized by the ShakuraSunyaev $\alpha$ parameter (Shakura \& Sunyaev 1973), which controls the transport properties of both dust and gas. The level of disk turbulence is difficult to constrain observationally: some estimates based on turbulent line broadening have suggested very low $\alpha$ values that then struggle to reproduce observed 
accretion rates (Flaherty et al. 2015), although the universality of this result has been questioned (e.g., Teague et al. 2016).

We explore hydrodynamical models in which $\alpha$ and the gas surface density are constrained by the observed high accretion rate onto the star $\left(\dot{M}=3 \times 10^{-8} M_{\odot} \mathrm{yr}^{-1}\right.$ McClure et al. 2013), assuming that this accretion is driven by some form of turbulent viscosity; moreover, the highly axisymmetric image implies that the disk is not self-gravitating. Together these constraints favor a rather high $\alpha$ value $\left(>10^{-2}\right)$. We also assume that the maximum grain size, $a_{\max }$, is locally determined by the minimum of two limits imposed by radial drift and fragmentation, assuming a fragmentation velocity of $10 \mathrm{~m} \mathrm{~s}^{-1}$ (Birnstiel et al. 2012). We compute the corresponding dust opacity (Tazzari et al. 2016), assuming a population of compact silicate grains with size distribution $d n / d a \propto a^{-3}$ for $a<a_{\max }$, and in our emissivity modeling adopt the temperature profile derived by Kwon et al. (2015). We assume an initial dust to gas ratio of 0.01 .

Below we describe simulations of our fiducial model with parameters $\alpha=0.014$ and total disk mass within 200 au of $20 M_{\text {Jupiter }}$. These parameters imply strong accretion in the disk $\left(\sim 10^{-8} M_{\odot} \mathrm{yr}^{-1}\right)$ and yield a profile of $a_{\max }$ that is compatible with measurements of the disk-averaged spectral index in CI Tau. ${ }^{9}$

We use these parameters in hydrodynamical simulations where we insert three planets in the disk, using the 2D version of the FARGO3D code (Benéz-Llambay \& Masset 2016) with our implementation of drag-coupled dust (Rosotti et al. 2016). We employ 350 logarithmically spaced cells in the radial direction (from 5.6 to $378 \mathrm{au}$ ) and 512 cells azimuthally, producing approximately square cells at each location. We adopt an initial gas surface density profile $\Sigma_{\text {gas }} \propto 1 / r$ normalized at $8 \mathrm{~g} \mathrm{~cm}^{-2}$ at $25 \mathrm{au}$, steepening to a $1 / r^{2}$ profile beyond $60 \mathrm{au}$. The local value of $a_{\max }$ is computed as above.

We then compute a synthetic emissivity profile (using the temperature profile and calculation of opacity as a function of $a_{\max }$ detailed above) for direct comparison with our GALARIO-derived profile. The purple dashed line in panel (d) of Figure 1 presents the brightness profile of our fiducial model, where planets of mass $0.75,0.15$ and $0.4 M_{\text {Jupiter }}$ are located at orbital radii of 14,43 , and 108 au. We also produce a synthesized image (Figure 2), generating model visibilities via the ft task in CASA with exactly the same $u v$-plane coverage and observational setup as the actual observations, and then CLEANing the image using the same imaging parameters as the observed image. Note that for a given disk model, the planet mass within the innermost gap is only determined to within around a factor of two because this gap is poorly resolved, while the values are constrained to within around $30 \%$ in the outer two gaps.

\section{Discussion}

\subsection{Observational Tests of the Fiducial Model}

Our fiducial model is motivated by reproducing accretion rate and spectral index data for CI Tau, which results in moderately high turbulence levels $(\alpha \sim 0.01)$. In HL Tau Pinte et al. (2016) argued for low turbulence levels on account of the narrowness of the ring features; in CI Tau, however, the somewhat wider gaps and colder disk means that the turbulence

\footnotetext{
9 A spectral index of $2.9 \pm 0.35$ is derived by comparing our $1.3 \mathrm{~mm}$ flux with the $2.7 \mathrm{~mm}$ measurements of Guilloteau et al. (2011).
}

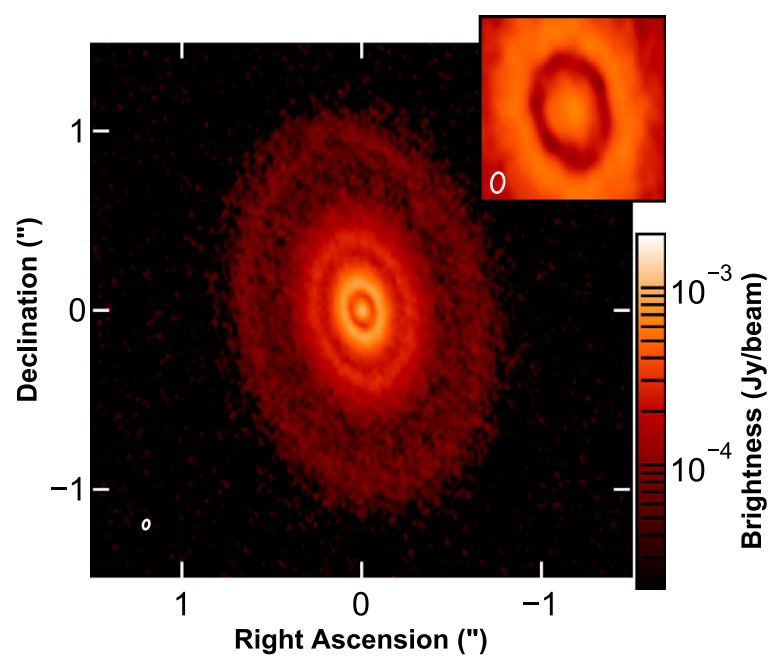

Figure 2. CLEANed image of the continuum emission obtained from our gas and dust hydrodynamical simulation containing three planets. This synthetic image was produced with the same noise level as in the observations and using the same imaging parameters used in Figure 1.

levels cannot be constrained in this way. The ratio of total fluxes at $2.7-1.3 \mathrm{~mm}$ in fact requires that $a_{\max }$ in the outer, optically thin regions of CI Tau is relatively low $(<1 \mathrm{~mm})$, in agreement with our model. CI Tau may be relatively unusual in lacking larger grains (its $\mathrm{mm}$ spectral index lies at the $\sim 85$ th percentile among protoplanetary disks; Testi et al. 2014). An alternative scenario, if we put aside the evidence from the $\mathrm{mm}$ spectral index for small grains in the outer disk, is that disk accretion is driven by a magnetized wind (e.g., Bai 2016) rather than turbulent viscosity. Low turbulence levels allow grains to grow and partially decouple from the flow so that lower planetary masses are required to match the observed gap parameters: from the hydrodynamical simulations of Rosotti et al. (2016; where $\alpha=10^{-3}$ ) we estimate planet masses of 20-30 earth masses for the outer two, although a mass of up to around a Jupiter mass can be accommodated in the case of the innermost planet. Spatially resolved spectral index determinations (Tazzari et al. 2016), as well as searches for possible kinematic distortions expected from a gas giant planet (Pinte et al. 2018; Teague et al. 2018), could potentially discriminate between these possibilities.

\subsection{Evolutionary Scenarios for the Fiducial Model: Formation and Migration}

The inferred planet masses in the three gaps suggest that none of these planets formed through gravitational instability. Planets formed in this way should exceed the Jeans limit in the outer disk (about a Jupiter mass) and should rapidly grow to much larger masses by accretion (Kratter \& Lodato 2016). The hot Jupiter, on the other hand, could have been formed by a variety of mechanisms; from the modeled masses in disk and planets and from the accretion onto the star, the inferred timescale for its inward migration is $\sim 0.4 \mathrm{Myr}$ (Dürmann \& Kley 2015). There would have been plenty of time for it to have migrated from a range of outward-lying locations.

The roughly Jovian mass planet inferred at 14 au is also easy to account for in terms of existing planet formation models (i.e., core accretion models involving either planetesimal or pebble accretion; Ida et al. 2013; Bitsch et al. 2015). However, 
neither of these models readily account for the two lower-mass planets at 43 and $108 \mathrm{au}$. The timescales for forming and accumulating solid material are long in the outer disk (though see Rafikov 2011 for arguments in favor of planetesimal accretion at large orbital radii). Even if this were circumvented, these planets would need to have grown through the mass range (10-20 earth masses) where rapid inward migration is expected (Paardekooper et al. 2011), and so their existence at large radii is a puzzle. Ida et al. (2013) were able to generate a modest population of gas giants at large separations through outward scattering of planetary cores and subsequent accretion, but their population synthesis models only sparsely populate the parameter space corresponding to the two outer planets in CI Tau.

It is unclear whether the current planetary architecture would survive on Gyr timescales. The planets' period ratios do not suggest a resonant configuration. Nevertheless, the relatively high disk mass means that they may still end up at small radii, possibly being swallowed by the star or ejected from the system by scattering off the hot Jupiter (Lega et al. 2013). While current imaging surveys of mature systems do not have the sensitivity to detect planets of the masses that we infer in CI Tau (Vigan et al. 2017), future surveys will be able to determine if CI Tau-like systems are long lived.

\subsection{Comparison with Other Gapped Disks}

High-resolution ALMA studies are steadily increasing the census of disks with annular substructure. Although unique in being the only such system with a hot Jupiter, CI Tau's ring structure is not unusual. Its well-spaced broad annuli place it in a similar category to HL Tau, HD 163296, and HD 169142 (ALMA Partnership et al. 2015; Isella et al. 2016; Fedele et al. 2017); none of the above systems exhibit the closely spaced shallow features seen in TW Hydra (Andrews et al. 2016) or the narrow deep features seen in AS 209 (Fedele et al. 2018). However, only TW Hydra and HL Tau have been observed at a comparably high resolution to this study. Previous modeling of the gap structures in HL Tau (e.g., Dipierro et al. 2015) have yielded similar planet masses as a function of disk parameters to what we report here, although in HL Tau the choice of disk model has not been constrained by other system observables.

\section{Conclusions}

High-resolution ALMA data of the disk in the young star CI Tau has revealed three prominent annular emission gaps which we have interpreted as an ensemble of massive planets spanning a factor 1000 in orbital radius. The wealth of supplementary data available on CI Tau has allowed us to construct models that are consistent with all of the data on this system available to date. The inferred planetary architecture suggests that the observed association between hot Jupiter and other companions may be in place at very early times. We note that the outer two planets (sub-Jovian planets at radii of 43 and $108 \mathrm{au}$ ) present a challenge to current planet formation models.

This work is supported by the DISCSIM project, grant agreement 341137 funded by the European Research Council under ERC-2013-ADG. M.K. acknowledges funding from the
European Unions Horizon 2020 program, Marie SklodowskaCurie grant agreement No. 753799, and F.M. from a Leverhulme Trust Early Career Fellowship, the Isaac Newton Trust and a Royal Society Dorothy Hodgkin Fellowship. This Letter uses ALMA data ADS/JAO.ALMA\#2016.1.01370.S. ALMA is a partnership of ESO (representing its member states), NSF (USA) and NINS (Japan), together with NRC (Canada) and NSC and ASIAA (Taiwan) and KASI (Republic of Korea), in cooperation with the Republic of Chile. The Joint ALMA Observatory is operated by ESO, AUI/NRAO and NAOJ.

Software: GALARIO v1.2 (Tazzari et al. 2018), emcee v2.2.0 (Foreman-Mackey et al. 2013), CASA v5.1.1 (McMullin et al. 2007).

\section{ORCID iDs}

C. J. Clarke (i) https://orcid.org/0000-0003-4288-0248

M. Tazzari $\odot$ https://orcid.org/0000-0003-3590-5814

S. Facchini (1) https://orcid.org/0000-0003-4689-2684

M. Kama (i) https://orcid.org/0000-0003-0065-7267

\section{References}

ALMA Partnership, Brogan, C. L., Pérez, L. M., et al. 2015, ApJL, 808, L3 Andrews, S. M., \& Williams, J. P. 2007, ApJ, 659, 705

Andrews, S. M., Wilner, D. J., Zhu, Z., et al. 2016, ApJL, 820, L40

Bai, X.-N. 2016, ApJ, 821, 80

Becker, J. C., Vanderburg, A., Adams, F. C., Khain, T., \& Bryan, M. 2017, AJ, 154, 230

Benéz-Llambay, P., \& Masset, F. S. 2016, ApJS, 223, 11

Biddle, L. I., Johns-Krull, C. M., Llama, J., Prato, L., \& Skiff, B. A. 2018, ApJL, 853, L34

Birnstiel, T., Klahr, H., \& Ercolano, B. 2012, A\&A, 539, A148

Bitsch, B., Lambrechts, M., \& Johansen, A. 2015, A\&A, 582, A112

Dipierro, G., Price, D., Laibe, G., et al. 2015, MNRAS, 453, L73

Dürmann, C., \& Kley, W. 2015, A\&A, 574, A52

Ercolano, B., Rosotti, G. P., Picogna, G., \& Testi, L. 2017, MNRAS, 464, L95

Fedele, D., Carney, M., Hogerheijde, M. R., et al. 2017, A\&A, 600, A72

Fedele, D., Tazzari, M., Booth, R., et al. 2018, A\&A, 610, A24

Flaherty, K. M., Hughes, A. M., Rosenfeld, K. A., et al. 2015, ApJ, 813, 99

Flock, M., Nelson, R. P., Turner, N. J., et al. 2017, ApJ, 850, 131

Flock, M., Ruge, J. P., Dzyurkevich, N., et al. 2015, A\&A, 574, A68

Foreman-Mackey, D., Hogg, D. W., Lang, D., \& Goodman, J. 2013, PASP, 125,306

Guilloteau, S., Dutrey, A., Piétu, V., \& Boehler, Y. 2011, A\&A, 529, A105

Guilloteau, S., Simon, M., Piétu, V., et al. 2014, A\&A, 567, A117

Haisch, K. E., Jr., Lada, E. A., \& Lada, C. J. 2001, ApJL, 553, L153

Ida, S., Lin, D. N. C., \& Nagasawa, M. 2013, ApJ, 775, 42

Isella, A., Guidi, G., Testi, L., et al. 2016, PhRvL, 117, 251101

Johns-Krull, C. M., McLane, J. N., Prato, L., et al. 2016, ApJ, 826, 206

Kley, W., \& Nelson, R. P. 2012, ARA\&A, 50, 211

Knutson, H. A., Fulton, B. J., Montet, B. T., et al. 2014, ApJ, 785, 126

Konishi, M., Hashimoto, J., \& Hori, Y. 2018, ApJL, 859, L28

Kratter, K., \& Lodato, G. 2016, ARA\&A, 54, 271

Kwon, W., Looney, L. W., Mundy, L. G., \& Welch, W. J. 2015, ApJ, 808, 102

Lega, E., Morbidelli, A., \& Nesvorný, D. 2013, MNRAS, 431, 3494

Mayor, M., \& Queloz, D. 1995, Natur, 378, 355

McClure, M. K., Calvet, N., Espaillat, C., et al. 2013, ApJ, 769, 73

McMullin, J. P., Waters, B., Schiebel, D., Young, W., \& Golap, K. 2007, in ASP Conf. Ser. 376, Astronomical Data Analysis Software and Systems XVI, ed. R. A. Shaw, F. Hill, \& D. J. Bell (San Francisco, CA: ASP), 127 Ngo, H., Knutson, H. A., Hinkley, S., et al. 2015, ApJ, 800, 138

Paardekooper, S.-J., Baruteau, C., \& Kley, W. 2011, MNRAS, 410, 293

Pinte, C., Dent, W. R. F., Ménard, F., et al. 2016, ApJ, 816, 25

Pinte, C., Price, D. J., Ménard, F., et al. 2018, ApJL, 860, L13 
Rafikov, R. R. 2011, ApJ, 727, 86

Rasio, F. A., \& Ford, E. B. 1996, Sci, 274, 954

Rosotti, G. P., Juhasz, A., Booth, R. A., \& Clarke, C. J. 2016, MNRAS, 459,2790

Shakura, N. I., \& Sunyaev, R. A. 1973, A\&A, 24, 337

Simon, M., Guilloteau, S., Di Folco, E., et al. 2017, ApJ, 844, 158

Tazzari, M., Beaujean, F., \& Testi, L. 2018, MNRAS, 476, 4527

Tazzari, M., Testi, L., Ercolano, B., et al. 2016, A\&A, 588, A53
Teague, R., Bae, J., Bergin, E. A., Birnstiel, T., \& Foreman-Mackey, D. 2018, ApJL, 860, L12

Teague, R., Guilloteau, S., Semenov, D., et al. 2016, A\&A, 592, A49

Testi, L., Birnstiel, T., Ricci, L., et al. 2014, in Protostars and Planets VI, ed. H. Beuther et al. (Tucson, AZ: Univ. Arizona Press), 339

Vigan, A., Bonavita, M., Biller, B., et al. 2017, A\&A, 603, A3

Wright, J. T., Marcy, G. W., Howard, A. W., et al. 2012, ApJ, 753, 160

Zhang, K., Bergin, E. A., Blake, G. A., et al. 2016, ApJL, 818, L16 